\title{
Simultaneous Determination of 11 Compounds in Gualou Guizhi Granule and Pharmacokinetics Study by UPLC-MS/MS
}

\author{
Chengtao Sun, ${ }^{1}$ Wen Xu, ${ }^{1,2}$ Yuqin Zhang, ${ }^{1}$ Lishuang Yu, ${ }^{1}$ Miao Ye, ${ }^{1,2}$ \\ Kedan Chu, ${ }^{1}$ Wei Xu, ${ }^{1}$ and Yu Lin ${ }^{1}$ \\ ${ }^{1}$ College of Pharmacy, Fujian University of Traditional Chinese Medicine, Fuzhou, Fujian 350122, China \\ ${ }^{2}$ Centre of Biomedical Research \& Development, Fujian University of Traditional Chinese Medicine, Fuzhou, Fujian 350122, China \\ Correspondence should be addressed to Wei Xu; xwflab@163.com and Yu Lin; lyfilab@163.com
}

Received 20 February 2017; Revised 16 May 2017; Accepted 25 May 2017; Published 20 July 2017

Academic Editor: Giuseppe Ruberto

Copyright (C) 2017 Chengtao Sun et al. This is an open access article distributed under the Creative Commons Attribution License, which permits unrestricted use, distribution, and reproduction in any medium, provided the original work is properly cited.

\begin{abstract}
A rapid and sensitive ultrafast performance liquid chromatography-tandem mass spectrometry method (UPLC-MS/MS) was developed for the simultaneous determination of 11 compounds in Gualou Guizhi Granule (GLGZG), including liquiritin, isoliquiritin, liquirtin apioside, isoliquiritin apioside, liquiritigenin, isoliquiritigenin, glycyrrhizic acid, glycyrrhetinic acid, paeoniflorin, albiflorin, and paeoniflorin sulfonate in rat plasma. UPLC-MS/MS assay with negative ion mode was performed on a Waters CORTECS C18 $(2.1 \times 100 \mathrm{~mm}, 1.6 \mu \mathrm{m})$ with the mobile phase consisting of $0.1 \%$ aqueous formic acid (A) and acetonitrile (B) in gradient elution at a flow rate of $0.25 \mathrm{~mL} \cdot \mathrm{min}^{-1}$. The method was linear for all analytes within the detection range $(r \geq 0.9597)$. The inter- and intraday precision (RSD) were 2.21-6.41\% and 1.67-6.18\%; the inter- and intraday accuracy (recover) were $92.48-114.03 \%$ and $90.23-112.04 \%$. And the recovery rate ranged from $81.30 \%$ to $108.22 \%$. The matrix effect values obtained for analytes ranged from $88.91 \%$ to $113.32 \%$. This validated method was successfully applied to a pharmacokinetics study in rats after oral administration of GLGZG.
\end{abstract}

\section{Introduction}

Prescription of Gualou Guizhi Granule (GLGZG) was first recorded in "Essentials from the Golden Cabinet" (around 210 AD) [1], which consisted of six herbs, including Trichosanthes kirilowii Maxim., Paeonia lactiflora Pall., Cinnamomum cassia Presl., Glycyrrhiza uralensis Fisch., Zingiber officinale Rosc., and Ziziphus jujuba Mill. according to yinyang and wuhsing (five elements) theory of Traditional Chinese Medicine (TCM) in a weight ratio of $10: 3: 3: 3: 2: 3$.

GLGZG has long been applied clinically to treat muscular spasticity following stroke, epilepsy, or spinal cord injury [24]. At present, the granule of GLGZG (Min drug system approval No. S20130001) has been approved to be a standard hospital prescription at Fujian University of TCM Affiliated Second People's Hospital (Fuzhou, China). In recent years, much attention has been paid to its phytochemical and biological studies. Phytochemical studies showed that
104 compounds in GLGZG were identified or tentatively characterized and several bioactive components, such as citrulline, luteolin, puerarin, liquiritin, taxifolin, naringin, formononetin, isoliquiritigenin, 6-gingerol, curcumin, caffeic acid, ferulic acid, jujuboside A, protocatechuic acid, cinnamic acid, catechin, and paeoniflorin, were quantified [5-7]. And pharmacological studies also showed that GLGZG had significant effects of anti-inflammatory [8-10], antioxidation [11], antiexcitotoxicity [12, 13], and antiapoptosis [14, $15]$ in vitro and in vivo. Meanwhile, to our knowledge, some literature about Trichosanthes kirilowii Maxim. [16, 17], protocatechuic acid [18], catechinic acid [19], curcumin [20], 6-gingerol [21], paeoniflorin [22], and isoliquiritigenin [23] proved that they had effect on cerebral ischemia-reperfusion injury.

However, pharmacokinetics (PK) of GLGZG has not been reported. Modern serum pharmacology researches think that figuring out the bioactive components of TCM and its 


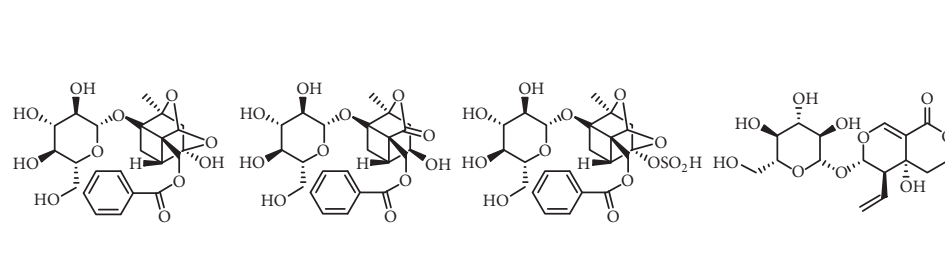

1

2
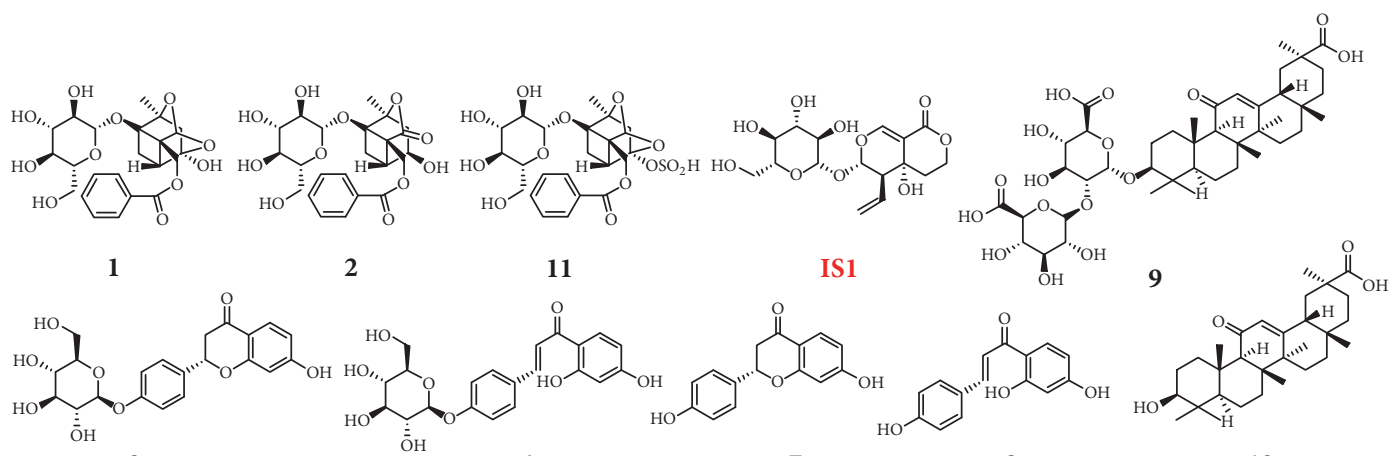

3

11

IS1

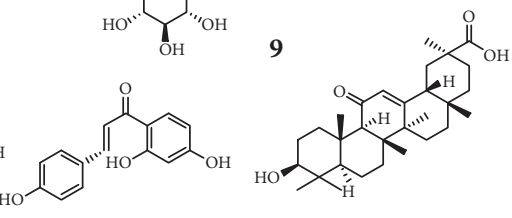

4
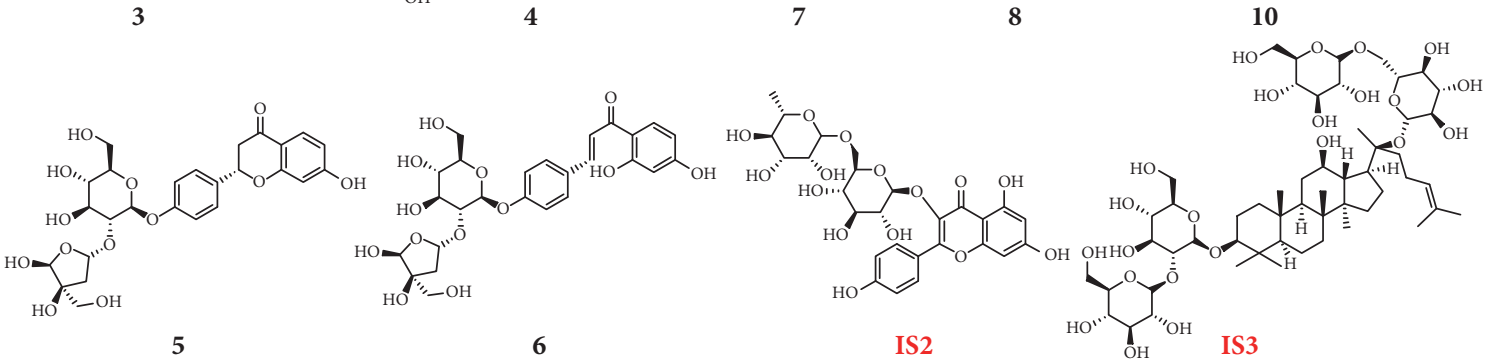

FIGURE 1: Chemical structures of the eleven standard reference compounds and ISs. 1: paeoniflorin; 2: albiflorin; 3: liquiritin; 4: isoliquiritin; 5: liquiritin apioside; 6: isoliquiritin apioside; 8: liquiritigenin; 9: isoliquiritigenin; 10: glycyrrhizic acid; 11: glycyrrhetinic acid; 12: paeoniflorin sulfonate; IS1: ginsenoside Rb1; IS2: swertiamarin; IS3: kaempferol-3-O-rutinoside.

chemical structural formula of metabolic product in human blood is extremely important [24]. Therefore, to support the comprehensive PK of GLGZG, it is important to develop a rapid and sensitive method to simultaneously determine the bioactive components in plasma samples. Our team's previous study showed that there were 42 ingredients including liquiritin, isoliquiritin, liquirtin apioside, isoliquiritin apioside, liquiritigenin, isoliquiritigenin, glycyrrhizic acid, glycyrrhetinic acid, paeoniflorin, albiflorin, and paeoniflorin sulfonate in rat plasma. Thus, above 11 ingredients were selected to assess the PK characteristics of GLGZG in this study $[5,6]$.

\section{Materials and Methods}

2.1. Materials. GLGZG were provided by Fujian University of TCM Affiliated Second People's Hospital (Fuzhou, China). Its voucher specimens were deposited in the College of Pharmacy, Fujian University of TCM. Standard substances (glycyrrhizic acid, glycyrrhetinic acid, isoliquiritigenin, and albiflorin) were purchased from Chengdu MUST Biological Technology Co., Ltd. (Sichuang, China), and standard substances (liquiritin, liquiritin apioside, and isoliquiritin apioside) and internal standards (swertiamarin, kaempferol3-O-rutinoside, and ginsenoside Rb1) were bought from the Shanghai Tauto Biotech Co., Ltd. (Shanghai, China). Paeoniflorin sulfonate was separated and purified by self, it is confirmed by MS and NMR and its purity was above 98\% by HPLC-DAD. Their structures were shown in Figure 1. Methanol and acetonitrile were of HPLC grade and purchased from Merck Co. (Darmstadt, Germany). All other chemical reagents evolved in this research were of analysis grade or better.

All specific pathogen-free (SPF), male Sprague-Dawley (SD) rats, weighing $250 \pm 20 \mathrm{~g}$, were bought from Beijing Weitonglihua Experimental Animal Technical Co., Ltd. (Beijing, China), animal qualified number SCXK (Jing) 20120001. The principles of laboratory animal care were followed and the study was approved by the Ethics Committee of Fujian University of TCM, China.

2.2. HPLC/MS/MS Conditions. Chromatographic separation was performed on an Waters CORTECS C18 $(2.1 \times 100 \mathrm{~mm}$, $1.6 \mu \mathrm{m})$ with the column temperature at $45^{\circ} \mathrm{C}$. The mobile phase was composed of $0.1 \%$ formic acid in water $(\mathrm{A})$ and acetonitrile (B) with a gradient program $(0-0.5 \mathrm{~min}$ from $8 \%$ to $10 \% \mathrm{~B} ; 0.5-2.5 \mathrm{~min}$ from $10 \%$ to $15 \% \mathrm{~B} ; 2.5-4.0 \mathrm{~min}$ from $15 \%$ to $60 \% \mathrm{~B} ; 4.0-5.0 \mathrm{~min}$ from $60 \%$ to $95 \% \mathrm{~B} ; 5.0-6.5 \mathrm{~min}$ $95 \% \mathrm{~B}$ ). The flow rate was $0.25 \mathrm{~mL} / \mathrm{min}$ and the injection volume was $1 \mu \mathrm{L}$.

Mass spectrometry was operated using a Waters (Milford, MA) Xevo TQMS with an electrospray ion source (ESI) in the negative ion multiple reaction monitoring (MRM) mode. The other detailed MS parameters were as follows: drying gas: $\mathrm{N}_{2}$ (purity of $99.9 \%$ ), $15 \mathrm{~L} / \mathrm{min}$, nebulizing gas: $50 \mathrm{~L} / \mathrm{min}$, capillary voltage: $2.5 \mathrm{kv}$. The optimized MRM parameters are summarized in Table 1.

2.3. Preparation of Standards, Internal Standard, and Quality Control (QC) Samples. Eleven standard stock solutions were prepared individually at concentrations $1 \mathrm{mg} / \mathrm{mL}$ by 
TABLE 1: MS analysis of parameters of detected compounds and internal standards (ISs).

\begin{tabular}{lccc}
\hline Analyte & RT (min) & {$[\mathrm{M}-\mathrm{H}]^{-}(\mathrm{m} / z)$} & $\mathrm{MS}^{n}(\mathrm{~m} / z)$ \\
\hline Paeoniflorin sulfonate (12) & 2.18 & 543.11 & 525.16 \\
Albiflorin (2) & 3.67 & 525.16 & 121.02 \\
Paeoniflorin (1) & 3.90 & 549.16 & 121.02 \\
Liquirtin apioside (5) & 4.04 & 417.12 & 255.06 \\
Liquiritin (3) & 4.07 & 549.16 & 255.06 \\
Isoliquiritin apioside (6) & 4.26 & 417.12 & 255.06 \\
Isoliquiritin (4) & 4.33 & 255.06 & 255.06 \\
Liquiritigenin (8) & 4.56 & 255.06 & 135.01 \\
Isoliquiritigenin (9) & 4.91 & 821.39 & 119.05 \\
Glycyrrhizic acid (10) & 5.02 & 469.33 & 351.05 \\
Glycyrrhetinic acid (11) & 6.22 & 1107.59 & 425.34 \\
Ginsenoside Rb1 (IS1) & 4.50 & 419.11 & 1107.59 \\
Swertiamarin (IS2) & 2.77 & 593.15 & 179.05 \\
Kaempferol-3-O-rutinoside (IS3) & 4.16 & & 285.04 \\
\hline
\end{tabular}

dissolving the substance in methanol. Then their working solutions were serially diluted with methanol with different concentrations used for plotting standard curves. Internal standards stock solution were also prepared in a concentration of $5 \mu \mathrm{g} \cdot \mathrm{mL}^{-1}$ for swertiamarin, $2.5 \mu \mathrm{g} \cdot \mathrm{mL}^{-1}$ for kaempferol-3-O-rutinoside, and $5 \mu \mathrm{g} \cdot \mathrm{mL}^{-1}$ for ginsenoside $\mathrm{Rb} 1$ in methanol. All the solutions were stored at $4^{\circ} \mathrm{C}$ until use.

The calibration curves and quality control (QC) samples were prepared by spiking the appropriate amount of working standards to blank rat plasma. The calibration curves were in the concentration of $0.04-8 \mu \mathrm{g} \cdot \mathrm{mL}^{-1}$ for paeoniflorin, $0.04-10 \mu \mathrm{g} \cdot \mathrm{mL}^{-1}$ for albiflorin, $0.005-1 \mu \mathrm{g} \cdot \mathrm{mL}^{-1}$ for liquiritin, $0.005-0.5 \mu \mathrm{g} \cdot \mathrm{mL}^{-1}$ for isoliquiritin, $0.005-0.5 \mu \mathrm{g} \cdot \mathrm{mL}^{-1}$ for liquirtin apioside, $0.005-0.5 \mu \mathrm{g} \cdot \mathrm{mL}^{-1}$ for isoliquiritin apioside, $0.001-0.2 \mu \mathrm{g} \cdot \mathrm{mL}^{-1}$ for liquiritigenin, $0.001-0.2 \mu \mathrm{g} \cdot \mathrm{mL}^{-1}$ for isoliquiritigenin, $\quad 0.05-10 \mu \mathrm{g} \cdot \mathrm{mL}^{-1}$ for glycyrrhizic acid, $0.01-4 \mu \mathrm{g} \cdot \mathrm{mL}^{-1}$ for glycyrrhetinic acid, and $0.05-$ $11.25 \mu \mathrm{g} \cdot \mathrm{mL}^{-1}$ for paeoniflorin sulfonate. QC samples were paeoniflorin $\left(0.1,2,8 \mu \mathrm{g} \cdot \mathrm{mL}^{-1}\right)$, albiflorin $\left(0.1,2,8 \mu \mathrm{g} \cdot \mathrm{mL}^{-1}\right)$, liquiritin $\left(0.01,0.2,0.5 \mu \mathrm{g} \cdot \mathrm{mL}^{-1}\right)$, isoliquiritin $(0.01,0.1$, $\left.0.25 \mu \mathrm{g} \cdot \mathrm{mL}^{-1}\right)$, liquirtin apioside $\left(0.01,0.1,0.25 \mu \mathrm{g} \cdot \mathrm{mL}^{-1}\right)$, isoliquiritin apioside $\left(0.01,0.1,0.25 \mu \mathrm{g} \cdot \mathrm{mL}^{-1}\right)$, liquiritigenin $\left(0.005, \quad 0.1, \quad 0.2 \mu \mathrm{g} \cdot \mathrm{mL}^{-1}\right)$, isoliquiritigenin $(0.005, \quad 0.02$, $\left.0.05 \mu \mathrm{g} \cdot \mathrm{mL}^{-1}\right)$, glycyrrhizic acid $\left(0.1,2,8 \mu \mathrm{g} \cdot \mathrm{mL}^{-1}\right)$, glycyrrhetinic acid $\left(0.01,1,2 \mu \mathrm{g} \cdot \mathrm{mL}^{-1}\right)$, and paeoniflorin sulfonate $\left(0.1,2,8 \mu \mathrm{g} \cdot \mathrm{mL}^{-1}\right)$.

2.4. Sample Preparation. Plasma samples (100 $\mu \mathrm{L}$ of plasma) and IS working solutions ( $10 \mu \mathrm{L}$ of swertiamarin, kaempferol3-O-rutinoside, and ginsenoside Rb1) were combined and briefly vortex-mixed for $3 \mathrm{~min}$ followed by the addition of 4 -fold acetonitrile. Then the mixture was centrifuged at $12000 \mathrm{rpm}$ for $10 \mathrm{~min}$. The supernatant was transferred to a clean tube and concentrated up to dryness; the residue was dissolved in $100 \mu \mathrm{L} 50 \%$ methanol. Finally, $1 \mu \mathrm{L}$ aliquot was injected into UPLC-MS/MS system for analysis.

\subsection{Method Validation}

2.5.1. Specificity. Specificity of the method was assessed by comparing chromatograms of six different sources of blank rat plasma with the corresponding spiked rat plasma. It was analyzed to investigate the presence of potential interferences from endogenous matrix components.

2.5.2. Linearity, Lower Limit of Quantification (LLOQ), and Lower Limit of Detection (LLOD). Linearity was evaluated on eight nonzero concentrations and was assessed by weighted $\left(1 / x^{2}\right)$ least-squares analysis. The LLOQ and LLOD were defined by signal-to-noise ratio method. LLOQ should be ten times the noise level $(\mathrm{S} / \mathrm{N} \geq 10)$ and LLOD should be three times the noise level $(S / N \geq 3)$. For each target constituent, the LLODs and LLOQs were determined by serial dilution of standard solution under the described UPLC-MS/MS conditions.

2.5.3. Precision and Accuracy. Intra- and interday variations were employed to evaluate the precision of the method. Intraday precision was evaluated in six replicates at three QC levels at one day and interday precision was evaluated at three QC levels at six days. The precision (RSD, \%) should not exceed $15 \%$ and the accuracy values should be within $15 \%$ of the actual values for QC samples.

2.5.4. Recovery and Matrix Effect. The recoveries of the analytes from plasma samples were determined by comparing the peak areas of the analytes in plasma samples after extraction to those of the same concentration of the analytes spiked into the solution extracted from blank plasma samples. The matrix effects were measured by comparing the peak 
TABLE 2: Calibration curves and linear ranges of detected compounds $(n=8)$.

\begin{tabular}{lccc}
\hline Analyte & Regression equation & $r$ & Linear range $(\mu \mathrm{g} / \mathrm{mL})$ \\
\hline Paeoniflorin & $y=0.381 x-0.009$ & $r=0.9980$ & $0.040-8.00$ \\
Albiflorin & $y=1.341 x-0.052$ & $r=0.9597$ & $0.040-10.00$ \\
Liquiritin & $y=112.3 x-1.519$ & $r=0.9930$ & $0.005-1.00$ \\
Isoliquiritin & $y=6.612 x-0.065$ & $r=0.9644$ & $0.005-0.50$ \\
Liquirtin apioside & $y=36.36 x-0.222$ & $r=0.9970$ & $0.005-0.50$ \\
Isoliquiritin apioside & $y=6.044 x-0.072$ & $r=0.9737$ & $0.005-0.50$ \\
Liquiritigenin & $y=123.0 x-1.408$ & $r=0.9910$ & $0.001-0.20$ \\
Isoliquiritigenin & $y=52.37 x-0.980$ & $r=0.9813$ & $0.001-0.20$ \\
Glycyrrhizic acid & $y=12.12 x-0.687$ & $r=0.9813$ & $0.050-1.00$ \\
Glycyrrhetinic acid & $y=2.443 x+0.019$ & $r=0.9985$ & $0.010-4.00$ \\
Paeoniflorin sulfonate & $y=7.091 x-0.112$ & $r=0.9985$ & $0.050-11.25$ \\
\hline
\end{tabular}

TABLE 3: LLOQ and LLOD of detected compounds.

\begin{tabular}{lccc}
\hline Analyte & $\begin{array}{c}\text { Linear range } \\
\left(\mu \mathrm{g} \cdot \mathrm{mL}^{-1}\right)\end{array}$ & $\begin{array}{c}\text { LLOQ } \\
\left(\mathrm{ng} \cdot \mathrm{mL}^{-1}\right)\end{array}$ & $\begin{array}{c}\text { LLOD } \\
\left(\mathrm{ng} \cdot \mathrm{mL}^{-1}\right)\end{array}$ \\
\hline Paeoniflorin & $0.04-8$ & 11.10 & 3.32 \\
Albiflorin & $0.04-10$ & 5.42 & 0.11 \\
Liquiritin & $0.005-1$ & 0.81 & 0.03 \\
Isoliquiritin & $0.005-0.5$ & 0.46 & 0.26 \\
Liquirtin apioside & $0.005-0.5$ & 0.88 & 0.14 \\
Isoliquiritin apioside & $0.005-0.5$ & 0.55 & 0.36 \\
Liquiritigenin & $0.001-0.2$ & 0.93 & 0.20 \\
Isoliquiritigenin & $0.001-0.2$ & 29.82 & 0.36 \\
Glycyrrhizic acid & $0.05-10$ & 15.74 & 10.67 \\
Glycyrrhetinic acid & $0.01-4$ & 4.21 & 5.95 \\
Paeoniflorin sulfonate & $0.05-11.25$ & & 1.52 \\
\hline
\end{tabular}

areas obtained from samples with the analytes spiked after extraction, at three QC concentration levels (low, middle, and high), to those obtained from standard solutions at the same concentrations.

2.5.5. Stability. Stability of the analytes from plasma samples was performed by determining three different concentrations (low, middle, and high samples) in six replicates under different conditions. It included short-term stability which was assessed by keeping samples at room temperature $\left(25^{\circ} \mathrm{C}\right)$ for $24 \mathrm{~h}$, stability which was measured using samples stored at $4^{\circ} \mathrm{C}$ for $24 \mathrm{~h}$, and freeze-thaw stability that was determined after three freeze-thaw cycles at $-80^{\circ} \mathrm{C}$.

2.6. Applications in Pharmacokinetics Studies. Six male SD rats, weighing $250 \pm 20 \mathrm{~g}$, were provided by Experimental Animal Research Center of Fujian TCM University (Fuzhou, China). And they were housed at controlled environmental conditions (temperature: $23 \pm 2^{\circ} \mathrm{C}$, relative humidity: 55 $\pm 10 \%)$ with free access to standard laboratory food and water. The rats were fasted for $12 \mathrm{~h}$ but with access to water before being given GLGZG $\left(3.6 \mathrm{~g} \cdot(\mathrm{kg} \cdot \mathrm{day})^{-1}\right)$. Blood samples of $0.3 \mathrm{~mL}$ were collected in heparin containing tubes from epicanthic veins of rats by capillary tube before dose $(0 \mathrm{~h})$ and at $5 \mathrm{~min}, 10 \mathrm{~min}, 20 \mathrm{~min}, 0.5 \mathrm{~h}, 1 \mathrm{~h}, 1.5 \mathrm{~h}, 2 \mathrm{~h}, 3 \mathrm{~h}, 6 \mathrm{~h}, 9 \mathrm{~h}$, and $12 \mathrm{~h}$. Then they were centrifuged at $12000 \mathrm{rpm}$ for $10 \mathrm{~min}$. The supernatant liquor was collected and frozen at $-20^{\circ} \mathrm{C}$ until use.

\section{Results and Discussion}

\subsection{Method Validation}

3.1.1. Specificity. Figure 2 was the typical chromatograms for blank plasma, blank plasma spiked 11 standard solutions, and IS working solutions. No obvious interferences from endogenous compound were found at the retention times and at the selected mass transitions of all the analytes and ISs.

3.1.2. Linearity, $L L O Q$, and $L L O D$. The regression equations, linear ranges, and correlation coefficients $(r)$ of eleven analytes were shown in Table 2. The calibration curves of eleven analytes exhibited good linearity. LLOQ and LLOD of eleven analytes were shown in Table 3. 

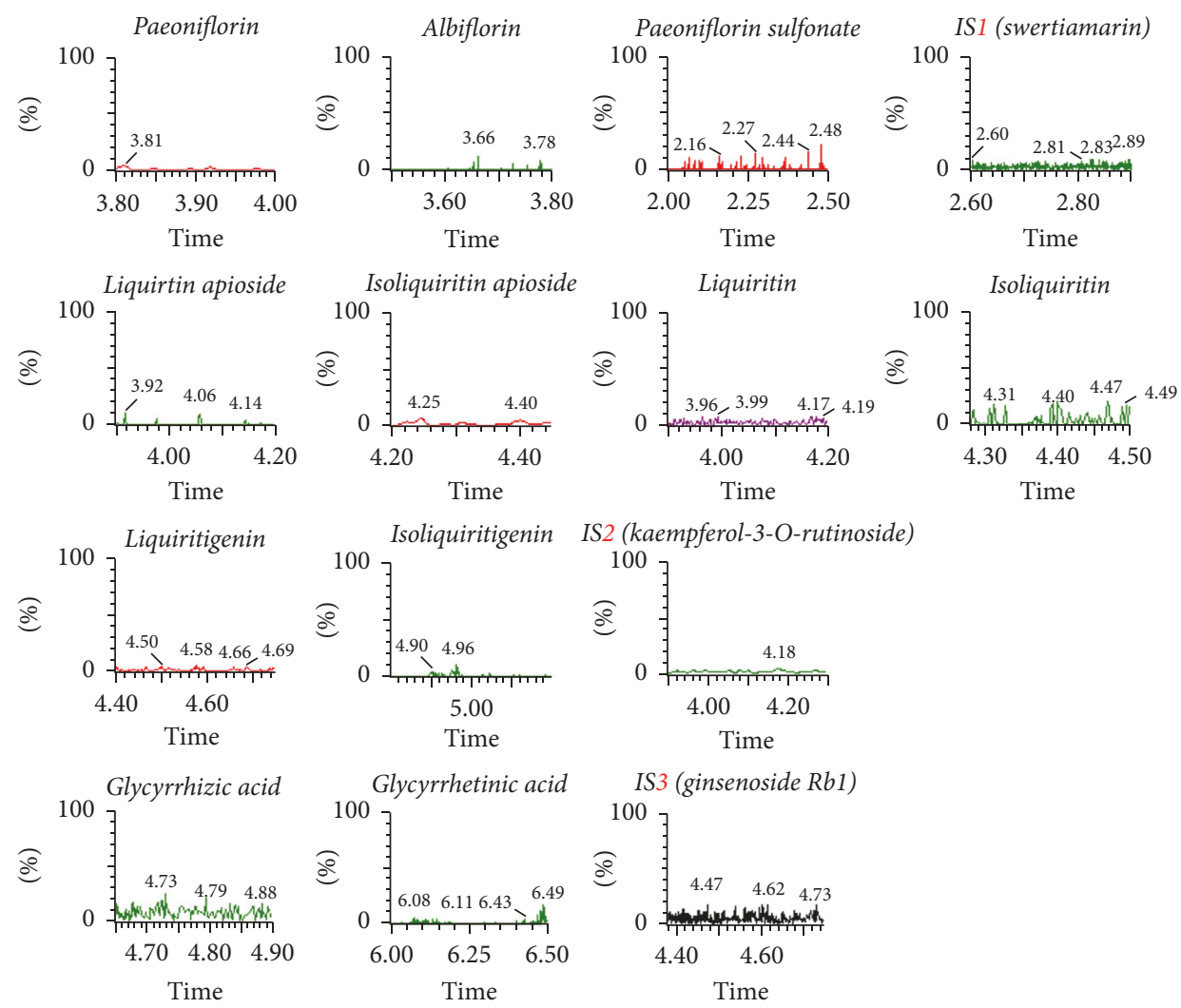

(a)
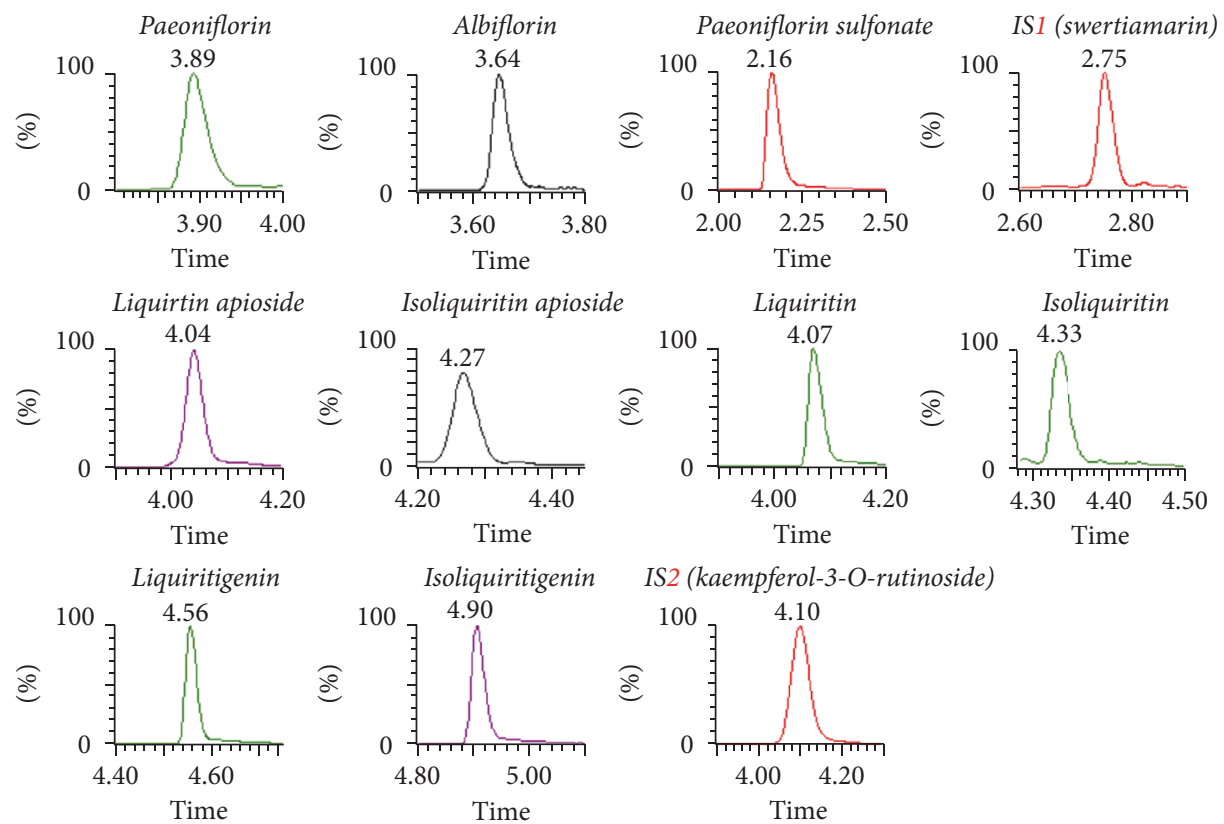

IS2 (kaempferol-3-O-rutinoside)
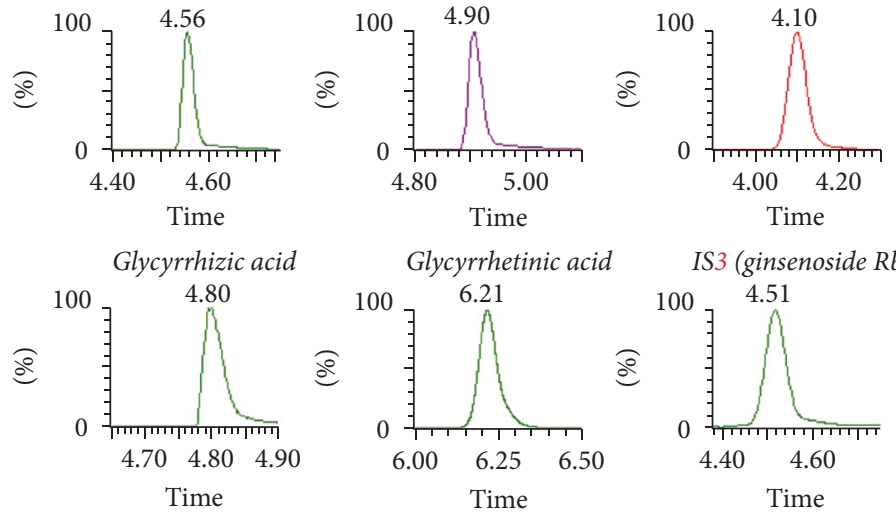

(b)

Figure 2: Continued. 

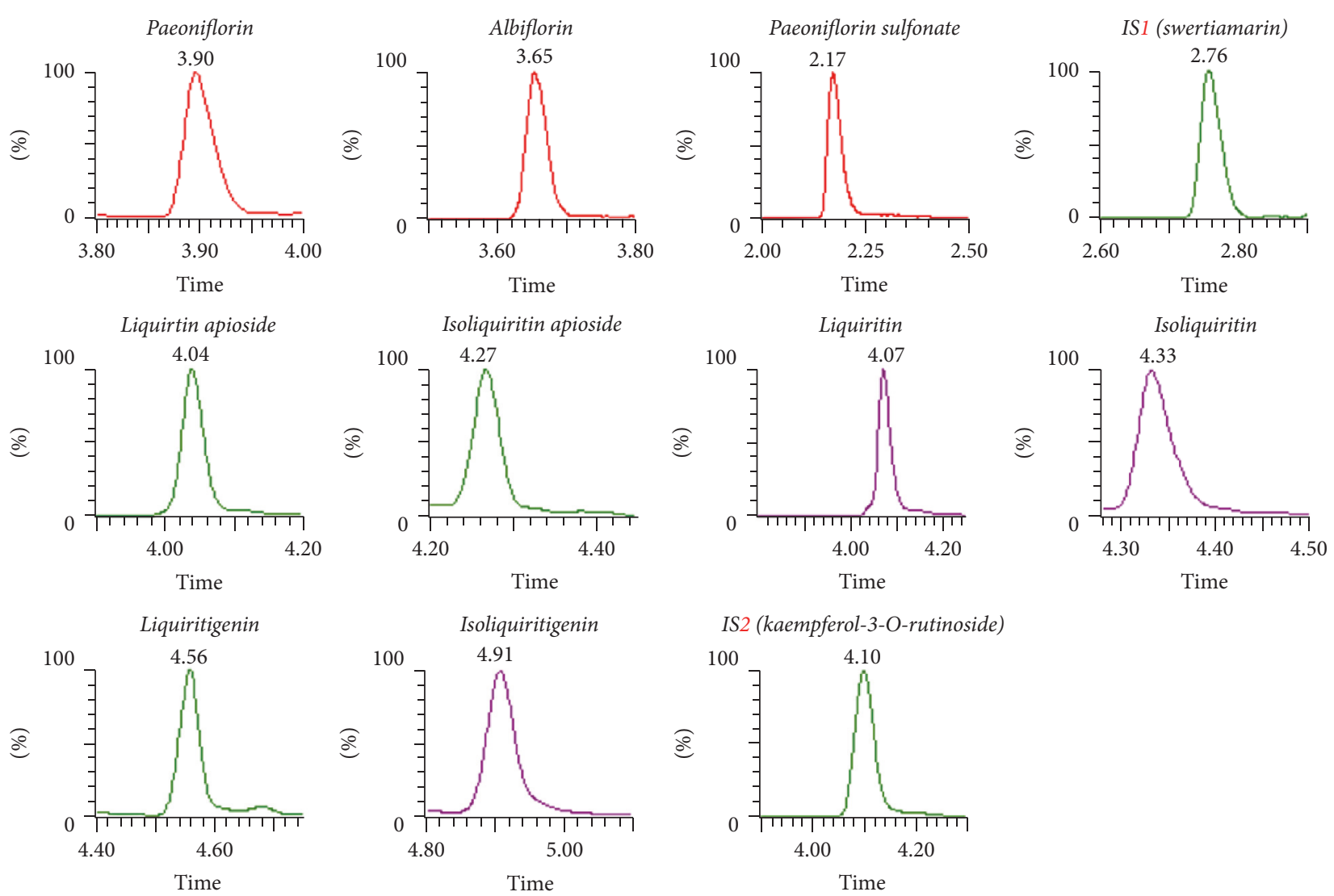

IS2 (kaempferol-3-O-rutinoside)
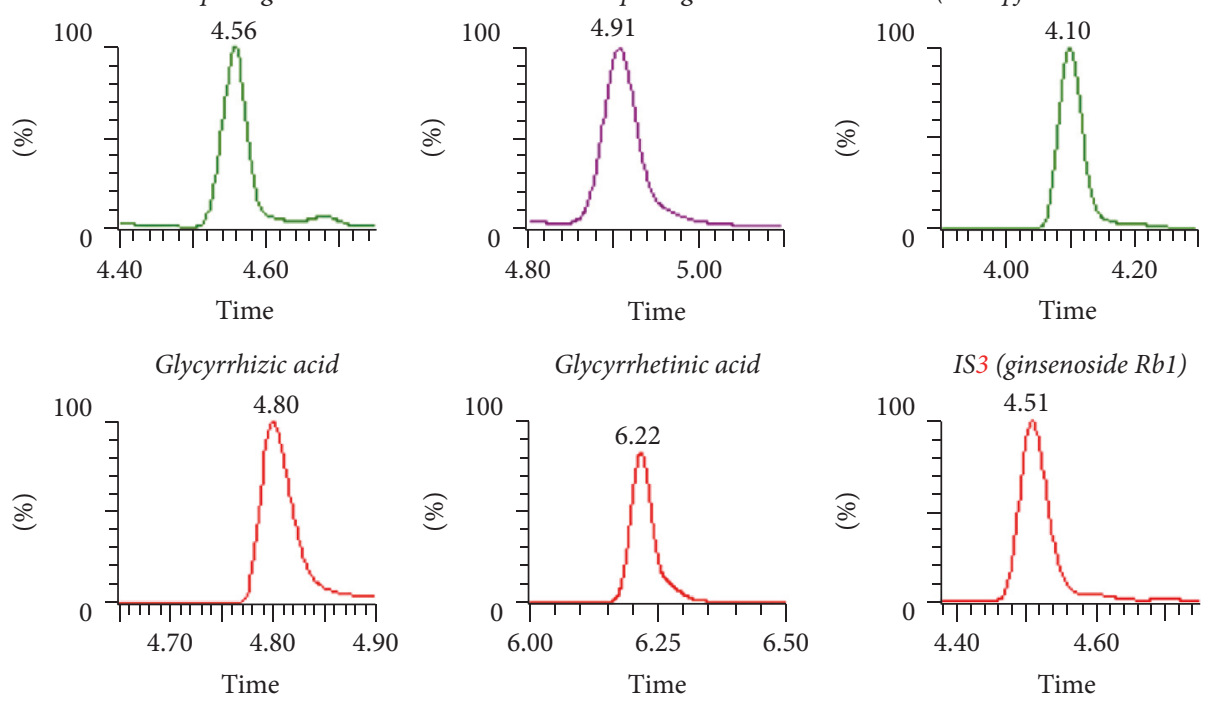

(c)

FIGURE 2: Typical MRM chromatograms GLGZG in rat plasma samples. (a) Blank rat plasma. (b) Plasma spiked with standards mix and IS. (c) Plasma sample after administration of GLGZG at $0.5 \mathrm{~h}$.

3.1.3. Precision and Accuracy. Results of the intra- and interday precision and accuracy were listed in Table 4 . The intraand interday precisions were $2.21-6.41 \%$ and $1.67-6.18 \%$. The intra- and interday accuracy were $92.48-114.03 \%$ and 90.23-112.04\%. It was found that, for all the analytes, both the accuracy and precision met the acceptable criterion.

3.1.4. Stability. Results of the stability tests were listed in Table 5. It was shown that all analytes were stable in plasma samples at room temperature $\left(25^{\circ} \mathrm{C}\right)$ for $24 \mathrm{~h}$, at $4^{\circ} \mathrm{C}$ in autosampler for $24 \mathrm{~h}$, and at $-80^{\circ} \mathrm{C}$ within three freeze-thaw cycles.

3.1.5. Recovery and Matrix Effect. Results of the extraction recoveries and matrix effects were summarized in Tables
6 and 7. The extraction recoveries of three concentration levels (low, middle, and high) QCs were 81.30-106.28\%, $85.14-108.22 \%$, and $88.65-104.21 \%$. It was indicated that the extraction recovery of this method was efficient, consistent, and reproducible. The matrix effect values obtained for all analytes at three concentration levels (low, middle, and high) QCs were 88.91-113.32\%, 91.78-107.86\%, and 96.87-110.07\%. There was no measurable matrix effect on the ionization of all analytes and ISs and this indicated that the method was acceptable.

3.2. Pharmacokinetic Study. The validated UPLC-MS/MS method was successfully applied to the PK studies of eleven analytes following oral administration of GLGZG to individual male rats $(n=6)$. The main pharmacokinetic 
TABLE 4: The precision and accuracy of detected compounds in rat plasma $(n=6)$.

\begin{tabular}{|c|c|c|c|c|c|}
\hline Analyte & $\begin{array}{c}\text { Nominal } \\
\text { concentration } \\
\left(\mu \mathrm{g} \cdot \mathrm{mL}^{-1}\right)\end{array}$ & $\begin{array}{l}\text { Intraday } \\
\text { precision } \\
\text { /RSD }\end{array}$ & $\begin{array}{l}\text { Interday } \\
\text { precision } \\
\text { /RSD }\end{array}$ & Intraday accuracy & Interday accuracy \\
\hline \multirow{3}{*}{ Paeoniflorin } & 0.1 & $4.98 \%$ & $5.52 \%$ & $97.35 \%$ & $96.93 \%$ \\
\hline & 2 & $4.44 \%$ & $3.33 \%$ & $92.48 \%$ & $92.08 \%$ \\
\hline & 8 & $3.77 \%$ & $3.46 \%$ & $107.09 \%$ & $106.62 \%$ \\
\hline \multirow{3}{*}{ Albiflorin } & 0.1 & $4.28 \%$ & $4.01 \%$ & $103.05 \%$ & $101.85 \%$ \\
\hline & 2 & $2.87 \%$ & $4.75 \%$ & $108.45 \%$ & $101.05 \%$ \\
\hline & 8 & $3.47 \%$ & $3.76 \%$ & $113.36 \%$ & $96.00 \%$ \\
\hline \multirow{3}{*}{ Liquiritin } & 0.01 & $5.53 \%$ & $4.68 \%$ & $99.15 \%$ & $104.48 \%$ \\
\hline & 0.2 & $5.28 \%$ & $3.62 \%$ & $94.19 \%$ & $109.29 \%$ \\
\hline & 0.5 & $3.32 \%$ & $4.31 \%$ & $97.90 \%$ & $105.66 \%$ \\
\hline \multirow{3}{*}{ Isoliquiritin } & 0.01 & $6.12 \%$ & $6.18 \%$ & $113.25 \%$ & $96.76 \%$ \\
\hline & 0.1 & $3.44 \%$ & $3.47 \%$ & $109.07 \%$ & $93.48 \%$ \\
\hline & 0.25 & $3.62 \%$ & $4.56 \%$ & $99.60 \%$ & $108.24 \%$ \\
\hline \multirow{3}{*}{ Liquirtin apioside } & 0.01 & $4.09 \%$ & $4.68 \%$ & $99.95 \%$ & $94.98 \%$ \\
\hline & 0.1 & $5.59 \%$ & $4.44 \%$ & $114.03 \%$ & $90.23 \%$ \\
\hline & 0.25 & $4.38 \%$ & $4.42 \%$ & $109.95 \%$ & $98.40 \%$ \\
\hline \multirow{3}{*}{ Isoliquiritin apioside } & 0.01 & $5.87 \%$ & $1.67 \%$ & $103.66 \%$ & $100.55 \%$ \\
\hline & 0.1 & $2.58 \%$ & $3.15 \%$ & $98.48 \%$ & $95.52 \%$ \\
\hline & 0.25 & $4.07 \%$ & $3.87 \%$ & $98.95 \%$ & $95.14 \%$ \\
\hline \multirow{3}{*}{ Liquiritigenin } & 0.005 & $4.62 \%$ & $4.01 \%$ & $94.95 \%$ & $96.74 \%$ \\
\hline & 0.1 & $4.87 \%$ & $5.13 \%$ & $94.62 \%$ & $94.19 \%$ \\
\hline & 0.2 & $2.57 \%$ & $5.14 \%$ & $109.56 \%$ & $106.41 \%$ \\
\hline \multirow{3}{*}{ Isoliquiritigenin } & 0.005 & $6.41 \%$ & $5.85 \%$ & $102.95 \%$ & $112.04 \%$ \\
\hline & 0.02 & $4.95 \%$ & $4.87 \%$ & $97.80 \%$ & $110.61 \%$ \\
\hline & 0.05 & $5.13 \%$ & $4.30 \%$ & $94.00 \%$ & $91.25 \%$ \\
\hline \multirow{3}{*}{ Glycyrrhizic acid } & 0.1 & $2.21 \%$ & $2.84 \%$ & $102.05 \%$ & $99.15 \%$ \\
\hline & 2 & $4.18 \%$ & $3.16 \%$ & $96.95 \%$ & $91.90 \%$ \\
\hline & 8 & $4.03 \%$ & $4.32 \%$ & $112.26 \%$ & $109.07 \%$ \\
\hline \multirow{3}{*}{ Glycyrrhetinic acid } & 0.01 & $3.58 \%$ & $4.01 \%$ & $98.59 \%$ & $100.15 \%$ \\
\hline & 1 & $5.88 \%$ & $4.31 \%$ & $108.85 \%$ & $111.16 \%$ \\
\hline & 2 & $2.97 \%$ & $5.19 \%$ & $92.82 \%$ & $110.17 \%$ \\
\hline \multirow{3}{*}{ Paeoniflorin sulfonate } & 0.1 & $3.90 \%$ & $5.85 \%$ & $97.71 \%$ & $99.35 \%$ \\
\hline & 2 & $3.54 \%$ & $4.72 \%$ & $93.66 \%$ & $94.38 \%$ \\
\hline & 8 & $3.62 \%$ & $5.15 \%$ & $107.48 \%$ & $96.05 \%$ \\
\hline
\end{tabular}

parameters were summarized in Table 8 and the mean plasma concentration-time profiles of the eleven analytes were illustrated in Figure 3.

As shown in Table 8 and Figure 3, the mean peak plasma time $\left(T_{\max }\right)$ of liquiritin, isoliquiritin, liquirtin apioside, isoliquiritin apioside, liquiritigenin, and isoliquiritigenin was $26.67 \mathrm{~min}-35.00 \mathrm{~min}$ after oral administration which suggested moderately rapid absorption pattern. Their area under the concentration-time and bioactivity-time curves (AUC) was calculated and ranged from $8.52 \mathrm{mg} \cdot(\mathrm{L} \cdot \mathrm{min})^{-1}$ to $54.87 \mathrm{mg} \cdot(\mathrm{L} \cdot \mathrm{min})^{-1}$. Moreover, rate of elimination of liquiritin was slow with the half-life $(t 1 / 2)$ values $309.46 \mathrm{~min}$. To our knowledge, the content of glycyrrhetinic acid was low but glycyrrhizic acid was high, and it was found that plasma concentration of glycyrrhetinic acid presented a trend of increasing; the phenomenon might be attributed to production 
TABLE 5: The stability of detected compounds in rat plasma $(n=6)$.

\begin{tabular}{|c|c|c|c|c|c|c|c|}
\hline \multirow[t]{2}{*}{ Analyte } & \multirow{2}{*}{$\begin{array}{c}\text { Nominal } \\
\text { concentration } \\
\left(\mu \mathrm{g} \cdot \mathrm{mL}^{-1}\right)\end{array}$} & \multicolumn{2}{|c|}{$\begin{array}{r}\text { Autosampler } \\
\text { stability }(24 \mathrm{~h})\end{array}$} & \multicolumn{2}{|c|}{$\begin{array}{c}\text { Stability } \\
(24 \mathrm{~h} \text { at room temperature })\end{array}$} & \multicolumn{2}{|c|}{$\begin{array}{l}\text { Freeze-thaw stability } \\
\qquad(3 \text { cycles })\end{array}$} \\
\hline & & Mean & RSD & Mean & RSD & Mean & RSD \\
\hline \multirow{3}{*}{ Paeoniflorin } & 0.1 & $103.46 \%$ & $5.06 \%$ & $105.66 \%$ & $2.67 \%$ & $97.71 \%$ & $2.01 \%$ \\
\hline & 2 & $98.29 \%$ & $5.07 \%$ & $91.25 \%$ & $4.29 \%$ & $92.82 \%$ & $4.30 \%$ \\
\hline & 8 & $113.81 \%$ & $3.60 \%$ & $109.75 \%$ & $3.44 \%$ & $107.48 \%$ & $3.77 \%$ \\
\hline \multirow{3}{*}{ Albiflorin } & 0.1 & $97.93 \%$ & $3.01 \%$ & $94.78 \%$ & $1.84 \%$ & $99.77 \%$ & $4.44 \%$ \\
\hline & 2 & $111.49 \%$ & $4.73 \%$ & $93.66 \%$ & $5.36 \%$ & $108.14 \%$ & $4.44 \%$ \\
\hline & 8 & $107.72 \%$ & $2.72 \%$ & $108.45 \%$ & $4.47 \%$ & $98.89 \%$ & $4.68 \%$ \\
\hline \multirow{3}{*}{ Liquiritin } & 0.01 & $112.48 \%$ & $3.87 \%$ & $95.70 \%$ & $4.18 \%$ & $101.75 \%$ & $5.02 \%$ \\
\hline & 0.2 & $92.30 \%$ & $3.83 \%$ & $110.81 \%$ & $3.68 \%$ & $109.75 \%$ & $4.11 \%$ \\
\hline & 0.5 & $90.84 \%$ & $5.13 \%$ & $106.73 \%$ & $2.84 \%$ & $97.91 \%$ & $3.02 \%$ \\
\hline \multirow{3}{*}{ Isoliquiritin } & 0.01 & $105.18 \%$ & $3.83 \%$ & $100.74 \%$ & $3.18 \%$ & $98.98 \%$ & $4.61 \%$ \\
\hline & 0.1 & $97.14 \%$ & $3.44 \%$ & $99.28 \%$ & $5.11 \%$ & $94.03 \%$ & $4.08 \%$ \\
\hline & 0.25 & $95.62 \%$ & $4.53 \%$ & $98.59 \%$ & $3.58 \%$ & $108.88 \%$ & $2.72 \%$ \\
\hline \multirow{3}{*}{ Liquirtin apioside } & 0.01 & $95.66 \%$ & $4.46 \%$ & $111.24 \%$ & $3.77 \%$ & $107.20 \%$ & $3.24 \%$ \\
\hline & 0.1 & $90.88 \%$ & $3.99 \%$ & $96.07 \%$ & $4.81 \%$ & $93.95 \%$ & $2.87 \%$ \\
\hline & 0.25 & $105.23 \%$ & $4.53 \%$ & $104.43 \%$ & $4.13 \%$ & $94.78 \%$ & $4.68 \%$ \\
\hline \multirow{3}{*}{ Isoliquiritin apioside } & 0.01 & $102.25 \%$ & $5.16 \%$ & $101.13 \%$ & $3.82 \%$ & $109.53 \%$ & $4.27 \%$ \\
\hline & 0.1 & $106.88 \%$ & $3.81 \%$ & $101.45 \%$ & $3.31 \%$ & $93.01 \%$ & $4.87 \%$ \\
\hline & 0.25 & $112.48 \%$ & $4.68 \%$ & $98.59 \%$ & $3.76 \%$ & $96.66 \%$ & $2.11 \%$ \\
\hline \multirow{3}{*}{ Liquiritigenin } & 0.005 & $98.45 \%$ & $3.58 \%$ & $111.60 \%$ & $4.41 \%$ & $97.45 \%$ & $6.59 \%$ \\
\hline & 0.1 & $93.53 \%$ & $5.60 \%$ & $108.45 \%$ & $4.01 \%$ & $93.29 \%$ & $5.43 \%$ \\
\hline & 0.2 & $97.16 \%$ & $3.17 \%$ & $97.03 \%$ & $3.73 \%$ & $108.02 \%$ & $4.68 \%$ \\
\hline \multirow{3}{*}{ Isoliquiritigenin } & 0.005 & $106.19 \%$ & $5.84 \%$ & $99.77 \%$ & $5.88 \%$ & $99.28 \%$ & $3.01 \%$ \\
\hline & 0.02 & $91.71 \%$ & $2.78 \%$ & $93.66 \%$ & $2.58 \%$ & $107.70 \%$ & $2.82 \%$ \\
\hline & 0.05 & $108.30 \%$ & $4.07 \%$ & $100.94 \%$ & $2.85 \%$ & $109.21 \%$ & $5.13 \%$ \\
\hline \multirow{3}{*}{ Glycyrrhizic acid } & 0.1 & $97.71 \%$ & $3.80 \%$ & $96.05 \%$ & $5.57 \%$ & $98.31 \%$ & $2.87 \%$ \\
\hline & 2 & $92.82 \%$ & $5.28 \%$ & $92.18 \%$ & $3.68 \%$ & $108.78 \%$ & $4.87 \%$ \\
\hline & 8 & $107.48 \%$ & $4.98 \%$ & $94.94 \%$ & $5.41 \%$ & $98.20 \%$ & $4.07 \%$ \\
\hline \multirow{3}{*}{ Glycyrrhetinic acid } & 0.01 & $102.25 \%$ & $3.35 \%$ & $111.03 \%$ & $4.54 \%$ & $111.93 \%$ & $4.95 \%$ \\
\hline & 1 & $97.14 \%$ & $3.34 \%$ & $95.89 \%$ & $3.72 \%$ & $92.58 \%$ & $2.44 \%$ \\
\hline & 2 & $96.54 \%$ & $5.64 \%$ & $90.19 \%$ & $4.81 \%$ & $94.32 \%$ & $3.02 \%$ \\
\hline \multirow{3}{*}{ Paeoniflorin sulfonate } & 0.1 & $102.25 \%$ & $4.44 \%$ & $109.21 \%$ & $2.23 \%$ & $99.57 \%$ & $6.05 \%$ \\
\hline & 2 & $96.28 \%$ & $4.30 \%$ & $94.32 \%$ & $5.67 \%$ & $94.59 \%$ & $4.82 \%$ \\
\hline & 8 & $93.03 \%$ & $5.43 \%$ & $96.38 \%$ & $2.25 \%$ & $93.39 \%$ & $5.13 \%$ \\
\hline
\end{tabular}

of glycyrrhetinic acid in the process of glycyrrhizic acid metabolism in vivo. Existing literature suggests that glycyrrhizic acid can be hydrolyzed to obtain the glycyrrhetinic acid in vivo by removing two molecules of glucuronic acid [25]. In addition, there was a double-peak phenomenon of glycyrrhizic acid, which may be because of reabsorption or enterohepatic circulation. This phenomena match the experimental result of a pharmacokinetic study of licorice, which was executed by Qiao et al. [26]. $T_{\max }$ of liquiritin is consistent with the study by Kamei et al. researchers [27] and it was found that isoliquiritigenin was low in the blood. In addition, in comparison with the related literatures, $T_{\max }$ and $C_{\max }$ 
TABLE 6: The extraction recovery of detected compounds in rat plasma $(n=6)$.

\begin{tabular}{|c|c|c|c|c|}
\hline \multirow{2}{*}{ Analyte } & \multirow{2}{*}{$\begin{array}{l}\text { Nominal concentration } \\
\left(\mu \mathrm{g} \cdot \mathrm{mL}^{-1}\right)\end{array}$} & \multicolumn{3}{|c|}{ Extraction recovery } \\
\hline & & Mean & SD & RSD \\
\hline \multirow{3}{*}{ Paeoniflorin } & 0.1 & $95.62 \%$ & $9.60 \%$ & $10.04 \%$ \\
\hline & 2 & $89.08 \%$ & $8.29 \%$ & $9.31 \%$ \\
\hline & 8 & $88.65 \%$ & $5.87 \%$ & $6.62 \%$ \\
\hline \multirow{3}{*}{ Albiflorin } & 0.1 & $95.43 \%$ & $8.97 \%$ & $9.40 \%$ \\
\hline & 2 & $95.33 \%$ & $10.93 \%$ & $11.47 \%$ \\
\hline & 8 & $92.33 \%$ & $8.32 \%$ & $9.01 \%$ \\
\hline \multirow{3}{*}{ Liquiritin } & 0.01 & $104.13 \%$ & $11.50 \%$ & $11.05 \%$ \\
\hline & 0.2 & $85.14 \%$ & $7.70 \%$ & $9.04 \%$ \\
\hline & 0.5 & $96.98 \%$ & $9.43 \%$ & $9.72 \%$ \\
\hline \multirow{3}{*}{ Isoliquiritin } & 0.01 & $90.56 \%$ & $7.54 \%$ & $8.32 \%$ \\
\hline & 0.1 & $86.10 \%$ & $8.39 \%$ & $9.74 \%$ \\
\hline & 0.25 & $91.48 \%$ & $4.68 \%$ & $5.12 \%$ \\
\hline \multirow{3}{*}{ Liquirtin apioside } & 0.01 & $90.38 \%$ & $8.90 \%$ & $9.85 \%$ \\
\hline & 0.1 & $99.47 \%$ & $6.93 \%$ & $6.96 \%$ \\
\hline & 0.25 & $90.76 \%$ & $10.23 \%$ & $11.27 \%$ \\
\hline \multirow{3}{*}{ Isoliquiritin apioside } & 0.01 & $81.30 \%$ & $7.70 \%$ & $9.47 \%$ \\
\hline & 0.1 & $108.22 \%$ & $7.52 \%$ & $6.95 \%$ \\
\hline & 0.25 & $93.61 \%$ & $9.43 \%$ & $10.07 \%$ \\
\hline \multirow{3}{*}{ Liquiritigenin } & 0.005 & $94.50 \%$ & $8.51 \%$ & $9.01 \%$ \\
\hline & 0.1 & $88.01 \%$ & $6.42 \%$ & $7.29 \%$ \\
\hline & 0.2 & $92.48 \%$ & $6.32 \%$ & $6.83 \%$ \\
\hline \multirow{3}{*}{ Isoliquiritigenin } & 0.005 & $102.31 \%$ & $9.49 \%$ & $9.27 \%$ \\
\hline & 0.02 & $101.35 \%$ & $9.76 \%$ & $9.63 \%$ \\
\hline & 0.05 & $90.08 \%$ & $5.19 \%$ & $5.76 \%$ \\
\hline \multirow{3}{*}{ Glycyrrhizic acid } & 0.1 & $93.11 \%$ & $8.21 \%$ & $8.82 \%$ \\
\hline & 2 & $100.35 \%$ & $9.58 \%$ & $9.54 \%$ \\
\hline & 8 & $99.46 \%$ & $6.38 \%$ & $6.41 \%$ \\
\hline \multirow{3}{*}{ Glycyrrhetinic acid } & 0.01 & $106.28 \%$ & $7.13 \%$ & $6.71 \%$ \\
\hline & 1 & $87.71 \%$ & $7.04 \%$ & $8.02 \%$ \\
\hline & 2 & $103.53 \%$ & $8.93 \%$ & $8.63 \%$ \\
\hline \multirow{3}{*}{ Paeoniflorin sulfonate } & 0.1 & $95.33 \%$ & $7.32 \%$ & $7.68 \%$ \\
\hline & 2 & $98.05 \%$ & $6.42 \%$ & $6.55 \%$ \\
\hline & 8 & $104.21 \%$ & $8.45 \%$ & $8.11 \%$ \\
\hline
\end{tabular}

of liquirtin apioside, isoliquiritin apioside, and paeoniflorin sulfonate were extended or shortened in different degrees. However, further studies should be carried out to verify these phenomena and assumptions, and the drug metabolism evaluation and the detailed pharmacokinetic/pharmacodynamic studies of GLGZG need more studies. The information described this paper might be helpful for further studies of GLGZG and beneficial for application in clinical therapy.

\section{Conclusion}

The UPLC-MS/MS method developed and validated in this study was specific and sensitive, with acceptable accuracy and a short run time of $7 \mathrm{~min}$ for the simultaneous determination of liquiritin, isoliquiritin, liquirtin apioside, isoliquiritin apioside, liquiritigenin, isoliquiritigenin, glycyrrhizic acid, glycyrrhetinic acid, paeoniflorin, albiflorin, and paeoniflorin 
TABLE 7: The matrix effect of detected compounds in rat plasma.

\begin{tabular}{|c|c|c|c|c|}
\hline \multirow{2}{*}{ Analyte } & \multirow{2}{*}{$\begin{array}{c}\text { Nominal concentration } \\
\left(\mu \mathrm{g} \cdot \mathrm{mL}^{-1}\right)\end{array}$} & \multicolumn{3}{|c|}{ Matrix effect } \\
\hline & & Mean & SD & RSD \\
\hline \multirow{3}{*}{ Paeoniflorin } & 0.1 & $104.05 \%$ & $4.16 \%$ & $4.00 \%$ \\
\hline & 2 & $93.79 \%$ & $5.89 \%$ & $6.28 \%$ \\
\hline & 8 & $98.33 \%$ & $6.01 \%$ & $6.11 \%$ \\
\hline \multirow{3}{*}{ Albiflorin } & 0.1 & $101.59 \%$ & $4.26 \%$ & $4.19 \%$ \\
\hline & 2 & $107.86 \%$ & $9.43 \%$ & $8.74 \%$ \\
\hline & 8 & $99.29 \%$ & $5.97 \%$ & $6.02 \%$ \\
\hline \multirow{3}{*}{ Liquiritin } & 0.01 & $88.91 \%$ & $6.93 \%$ & $7.79 \%$ \\
\hline & 0.2 & $106.80 \%$ & $7.42 \%$ & $6.95 \%$ \\
\hline & 0.5 & $102.29 \%$ & $5.04 \%$ & $4.92 \%$ \\
\hline \multirow{3}{*}{ Isoliquiritin } & 0.01 & $97.40 \%$ & $5.23 \%$ & $5.37 \%$ \\
\hline & 0.1 & $91.78 \%$ & $4.15 \%$ & $4.52 \%$ \\
\hline & 0.25 & $98.38 \%$ & $9.32 \%$ & $9.48 \%$ \\
\hline \multirow{3}{*}{ Liquirtin apioside } & 0.01 & $113.32 \%$ & $5.30 \%$ & $4.68 \%$ \\
\hline & 0.1 & $105.87 \%$ & $6.20 \%$ & $5.86 \%$ \\
\hline & 0.25 & $99.60 \%$ & $5.64 \%$ & $5.66 \%$ \\
\hline \multirow{3}{*}{ Isoliquiritin apioside } & 0.01 & $110.04 \%$ & $3.08 \%$ & $2.80 \%$ \\
\hline & 0.1 & $106.10 \%$ & $3.46 \%$ & $3.27 \%$ \\
\hline & 0.25 & $100.67 \%$ & $4.21 \%$ & $4.18 \%$ \\
\hline \multirow{3}{*}{ Liquiritigenin } & 0.005 & $89.86 \%$ & $6.43 \%$ & $7.16 \%$ \\
\hline & 0.1 & $94.92 \%$ & $6.30 \%$ & $6.64 \%$ \\
\hline & 0.2 & $99.45 \%$ & $6.61 \%$ & $6.65 \%$ \\
\hline \multirow{3}{*}{ Isoliquiritigenin } & 0.005 & $108.12 \%$ & $7.51 \%$ & $6.94 \%$ \\
\hline & 0.02 & $101.51 \%$ & $8.30 \%$ & $8.18 \%$ \\
\hline & 0.05 & $96.87 \%$ & $4.63 \%$ & $4.78 \%$ \\
\hline \multirow{3}{*}{ Glycyrrhizic acid } & 0.1 & $99.71 \%$ & $8.42 \%$ & $8.44 \%$ \\
\hline & 2 & $90.76 \%$ & $8.72 \%$ & $9.61 \%$ \\
\hline & 8 & $106.96 \%$ & $8.42 \%$ & $7.87 \%$ \\
\hline \multirow{3}{*}{ Glycyrrhetinic acid } & 0.01 & $95.51 \%$ & $6.25 \%$ & $6.55 \%$ \\
\hline & 1 & $93.47 \%$ & $4.64 \%$ & $4.97 \%$ \\
\hline & 2 & $101.34 \%$ & $5.93 \%$ & $5.85 \%$ \\
\hline \multirow{3}{*}{ Paeoniflorin sulfonate } & 0.1 & $100.75 \%$ & $8.54 \%$ & $8.48 \%$ \\
\hline & 2 & $104.38 \%$ & $7.23 \%$ & $6.93 \%$ \\
\hline & 8 & $110.07 \%$ & $6.31 \%$ & $5.73 \%$ \\
\hline
\end{tabular}

TABLE 8: Pharmacokinetic parameters of 11 compounds in rat plasma in Gualou Guizhi decoction $(n=6)$.

\begin{tabular}{lcccccc}
\hline Analyte & $\begin{array}{c}C_{\max } \\
\left(\mathrm{mg} \cdot \mathrm{mL}^{-1}\right)\end{array}$ & $\begin{array}{c}T_{\max } \\
(\mathrm{min})\end{array}$ & $\begin{array}{c}T_{1 / 2 z} \\
(\mathrm{~min})\end{array}$ & $\begin{array}{c}\mathrm{AUC}_{(0-\infty)} \\
\left(\mathrm{mg} \cdot(\mathrm{L} \cdot \mathrm{min})^{-1}\right)\end{array}$ & $\begin{array}{c}\mathrm{MRT}_{(0-\infty)} \\
(\mathrm{min})\end{array}$ & $\begin{array}{c}V z / F \\
(\mathrm{~L} \cdot \mathrm{kg}\end{array}$ \\
\hline Paeoniflorin & $4.16 \pm 0.72$ & $80.00 \pm 17.32$ & $142.98 \pm 30.11$ & $904.73 \pm 317.76$ & $235.91 \pm 40.45$ & $6.31 \pm 1.63$ \\
Albiflorin & $6.41 \pm 2.01$ & $60.00 \pm 21.21$ & $127.68 \pm 35.74$ & $1263.37 \pm 306.91$ & $215.15 \pm 45.14$ & $2.67 \pm 0.87$ \\
Liquiritin & $0.41 \pm 0.09$ & $30.00 \pm 0.00$ & $309.46 \pm 66.39$ & $54.87 \pm 14.40$ & $355.95 \pm 72.55$ & $167.40 \pm 35.86$ \\
Isoliquiritin & $0.15 \pm 0.04$ & $28.33 \pm 4.08$ & $276.19 \pm 51.94$ & $27.17 \pm 2.69$ & $339.62 \pm 46.83$ & $297.43 \pm 70.05$ \\
Liquirtin apioside & $0.23 \pm 0.06$ & $30.00 \pm 0.00$ & $234.63 \pm 60.70$ & $44.70 \pm 9.12$ & $319.49 \pm 61.75$ & $152.93 \pm 31.84$ \\
Isoliquiritin apioside & $0.11 \pm 0.02$ & $35.00 \pm 12.25$ & $244.62 \pm 34.16$ & $18.90 \pm 3.20$ & $315.50 \pm 43.96$ & $378.57 \pm 56.73$ \\
Liquiritigenin & $0.12 \pm 0.03$ & $26.67 \pm 5.16$ & $232.26 \pm 28.65$ & $20.38 \pm 4.30$ & $295.11 \pm 25.08$ & $340.80 \pm 73.56$ \\
Isoliquiritigenin & $0.04 \pm 0.01$ & $30.00 \pm 0.00$ & $203.86 \pm 40.50$ & $8.52 \pm 0.98$ & $285.44 \pm 50.94$ & $700.08 \pm 187.21$ \\
Glycyrrhizic acid & $4.37 \pm 1.49$ & $54.00 \pm 13.42$ & $171.54 \pm 33.67$ & $1262.61 \pm 158.73$ & $264.02 \pm 49.72$ & $3.92 \pm 1.44$ \\
Glycyrrhetinic acid & $2.92 \pm 0.67$ & $600 \pm 146.97$ & $132.11 \pm 28.33$ & $2041.10 \pm 706.58$ & $709.84 \pm 83.32$ & $2.91 \pm 1.44$ \\
Paeoniflorin sulfonate & $4.50 \pm 1.06$ & $80.00 \pm 30.11$ & $142.98 \pm 30.11$ & $777.42 \pm 163.06$ & $235.91 \pm 40.45$ & $6.31 \pm 1.63$ \\
\hline
\end{tabular}



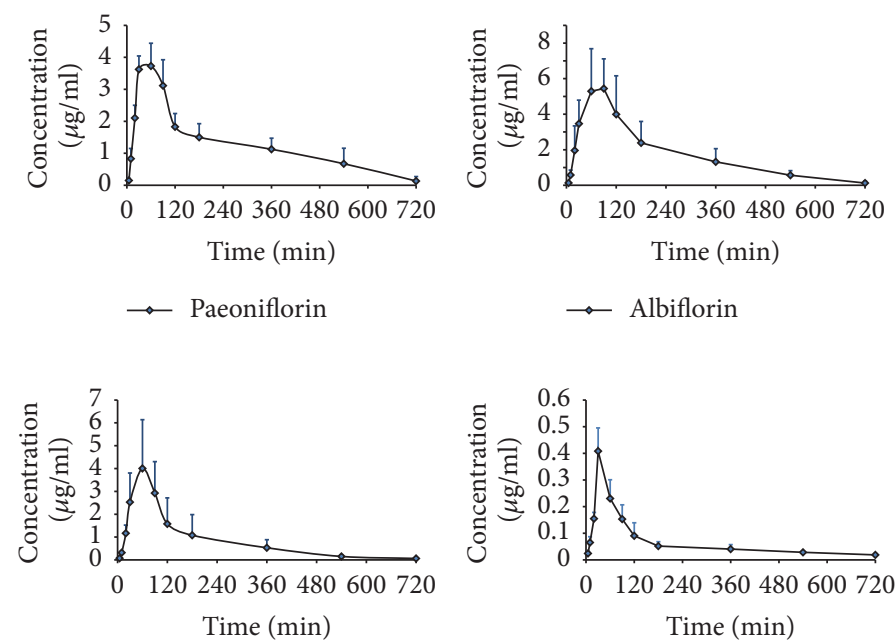

$\rightarrow$ Paeoniflorin sulfurous
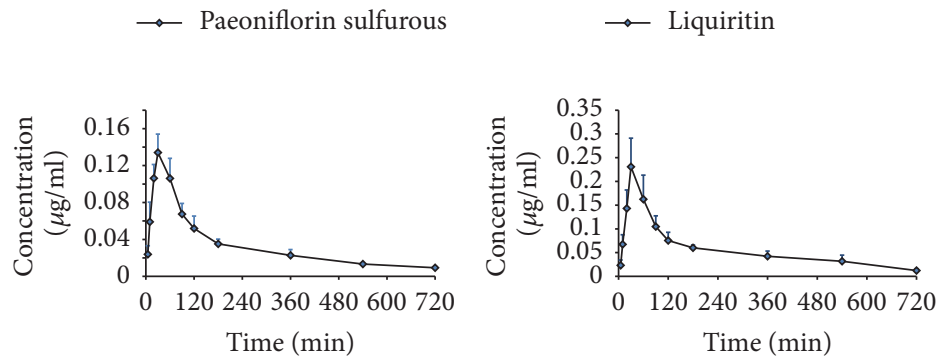

$\rightarrow$ Isoliquiritin
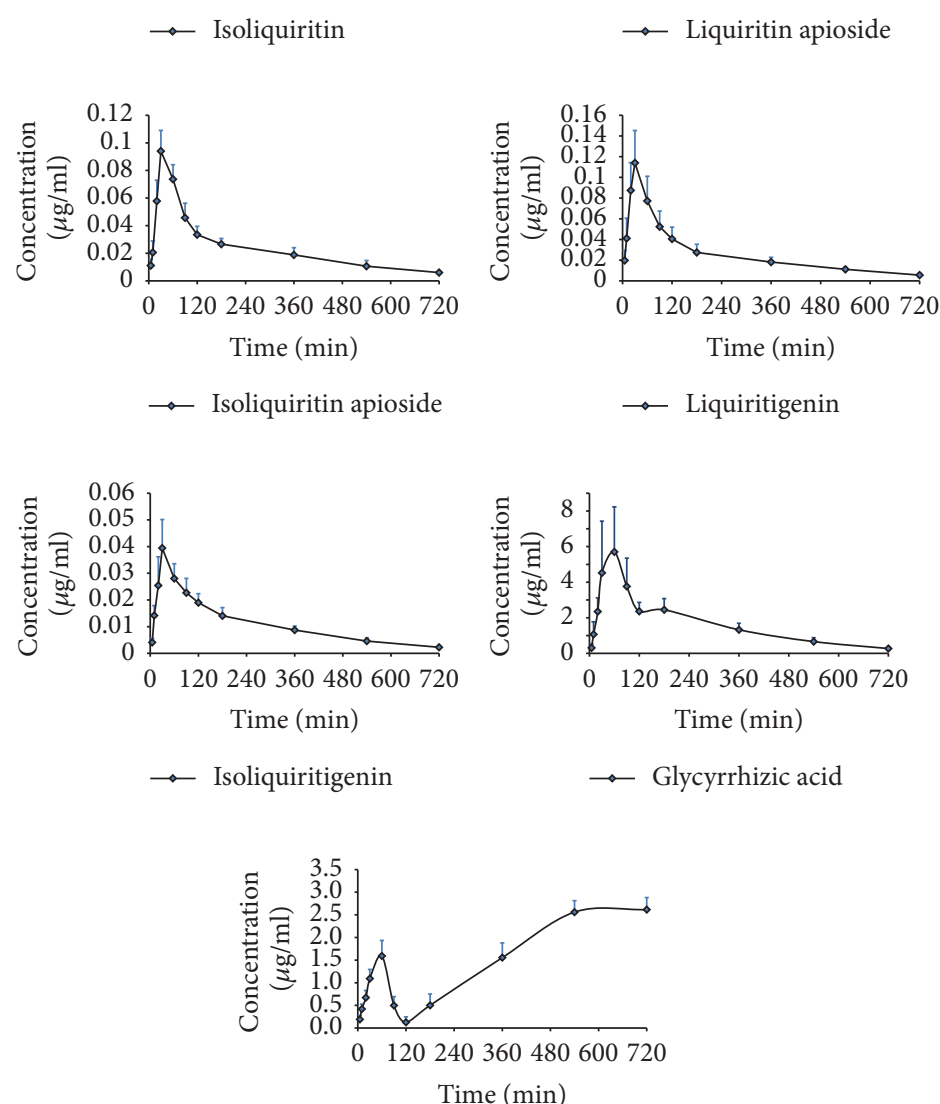

$\rightarrow$ Glycyrrhetinic acid

FIGURE 3: Concentration-time curve of 11 detected compounds in rats. 
sulfonate together in vivo following the oral administration of GLGZG. The results might be helpful to provide certain references to clinical application of this medicine or others.

\section{Conflicts of Interest}

The authors declare no conflicts of interest.

\section{Acknowledgments}

This research was done in State Key Laboratory of Chinese Pharmacies of Fujian Provincial Department of Science and Technology, Collaborative Innovation Center for Rehabilitation Technology, and TCM Rehabilitation Research Center of SATCM. It was supported by Project of Fujian Province Colleges and Universities in the New Century Excellent Talents, 2015 Strategic Emerging Industries Project of Fujian Province (no. 2015Y0067), and National Natural Science Foundation of China (no. 81503204 and no. 81674046).

\section{References}

[1] Z. Zhang, Y. Jinkui, Y. Lin, P. Yang, X. Hou, and Y. Yang, Synopsis of Golden Chamber, Macmillan Press, Beijing, China, 2008.

[2] X. Sun, "Research on formula treating paralysis and spasticity," in Treatise on Febrile and Miscellaneous Diseases, vol. 8, pp. 644645, Zhongguo Zhong Yi Ji Chu Yi Xue Za Zhi, 8, 2010.

[3] L. Zhang and H. Ai, "Effects of Gualou Guizhi decoction on cfos and c-jun in epileptic rats," Sichuan Huaxi Zhong Yi Yao Yan Jiu Suo, vol. 23, pp. 21-22, 2005.

[4] C. Yang, L. Chen, and J. Tao, "New usage of a classical formulaGua Lou GuiZhi decoction," Liaoning Zhong Yi Za Zhi, vol. 8, pp. 166-167, 2010.

[5] W. Xu, M. Q. Huang, H. Li et al., "Chemical profiling and quantification of Gua-Lou-Gui-Zhi decoction by high performance liquid chromatography/quadrupole-time-of-flight mass spectrometry and ultra-performance liquid chromatography/triple quadrupole mass spectrometry," Journal of Chromatography. B, Analytical Technologies in the Biomedical and Life Sciences, vol. 1, pp. 69-84, 986-987, 2015.

[6] Y. Lin, W. Xu, M. Q. Huang, Y. Q. Zhang, H. Li, and K. D. Chu $\mathrm{L}$, "Simultaneous determination of 41 components in Gualou Guizhi granules by UPLC coupled with triple quadrupole mass spectrometry," Analytical Methods, vol. 7, no. 2, pp. 8285-8296, 2015.

[7] X. W. Chen, H. Li, L. D. Chen et al., "Simultaneous determination of six active ingredients in Gualou Guizhi decoction by HPLC," Journal of Fujian University of Traditional Chinese Medicine, vol. 24, no. 2, pp. 31-34, 2014.

[8] Y. Zhang, S. Zhang, H. Li et al., "Ameliorative effects of Gualou Guizhi decoction on inflammation in focal cerebral ischemicreperfusion injury," Molecular Medicine Reports, vol. 12, no. 1, pp. 988-994, 2015.

[9] H. Hu, Z. Li, X. Zhu et al., "GuaLou GuiZhi decoction inhibits LPS-induced microglial cell motility through the MAPK signaling pathway," International Journal of Molecular Medicine, vol. 32, no. 6, pp. 1281-1286, 2013.

[10] H. Hu, Z. Li, X. Zhu et al., "Gua Lou Gui Zhi decoction suppresses LPS-induced activation of the TLR4/NF- $\kappa$ B pathway in BV-2 murine microglial cells," International Journal of Molecular Medicine, vol. 31, no. 6, pp. 1327-1332, 2013.
[11] S. Zhang, Y. Zhang, H. Li et al., "Antioxidant and antiexcitotoxicity effect of Gualou Guizhi decoction on cerebral ischemia/reperfusion injury in rats," Experimental and Therapeutic Medicine, vol. 9, no. 6, pp. 2121-2126, 2015.

[12] X. Chen, H. Li, M. Huang et al., "Effect of Gua Lou Gui Zhi decoction on focal cerebral ischemia-reperfusion injury through regulating the expression of excitatory amino acids and their receptors," Molecular Medicine Reports, vol. 10, no. 1, pp. 248-254, 2014.

[13] J. Huang, J. Tao, X. Xue et al., "Gua Lou Gui Zhi decoction exerts neuroprotective effects on post-stroke spasticity via the modulation of glutamate levels and AMPA receptor expression," International Journal of Molecular Medicine, vol. 31, no. 4, pp. 841-848, 2013.

[14] Y. Zhang, H. Li, M. Huang et al., "Neuroprotective effects of Gualou Guizhi decoction in vivo and in vitro," Journal of Ethnopharmacology, pp. 76-84, 2014.

[15] Y. Lin, W. Xu, Y. Q. Zhang, and H. Li, "Anti-apoptosis effects of Gualou Guizhi granule on stroke rats and hippocampal neuron," Journal of Rehabilitation Medicine, vol. 25, no. 1, pp. 38-43, 2015.

[16] W. Chen, H. Wang, and S. W. Zhang, "Effect of trichosanthin on High Plasma Homocystein in rats with cerebral ischemiareperfusion," JETCM, vol. 20, no. 2, pp. 275-277, 2011.

[17] W. Chen, S. W. Zhang, and H. Wang, "Study of radices trichosanthis on neuronal apoptosisin rats with local cerebral ischemia reperfusion," Modern Journal of Integrated Traditional Chinese, vol. 20, no. 15, pp. 1844-1845, 2011.

[18] S. Li, Z. Shi, H. Zhang et al., "Assessing gait impairment after permanent middle cerebral artery occlusion in rats using an automated computer-aided control system," Behavioural Brain Research, vol. 250, pp. 174-191, 2013.

[19] S. L. Liu, Z. W. Liu, P. Q. Lu et al., "Protective effects of catechin on cerebral ischemia-reperfusion injury in rats and its mechanism," Chinese Pharmacological Bulletin, vol. 26, no. 2, pp. 255-257, 2010.

[20] J. R. Lei, J. Qin, J. Zhang, K. Huang, M. R. Fu, and Z. M. Zhou, "Effects of curcumin on inflammatory reaction and blood-brain barrier permeability in rats following cerebral ischemic injury," Chinese Pharmacological Bulletin, vol. 26, no. 1, pp. 120-123, 2010.

[21] Q. Jiang and B. Xia, "The anti-inflammatory effect of 6gingerol on focal ischemia-reperfusion injury in rats," Journal of Xiangningcolledge (Medical Sciences), vol. 21, no. 4, pp. 281283, 2007.

[22] R.-B. Guo, G.-F. Wang, A.-P. Zhao, J. Gu, X.-L. Sun, and G. Hu, "Paeoniflorin protects against ischemia-induced brain damages in rats via inhibiting MAPKs/NF- $\kappa$ B-mediated inflammatory responses," PLoS ONE, vol. 7, no. 11, Article ID e49701, 2012.

[23] C. Zhan and J. Yang, "Protective effects of isoliquiritigenin in transient middle cerebral artery occlusion-induced focal cerebral ischemia in rats," Pharmacological Research, vol. 53, no. 3, pp. 303-309, 2006.

[24] X. J. Wang, "Studies on serum pharmacochemistry of traditional chinese medicine," World Science and Technology, vol. 4, no. 02, pp. 14-78, 2002.

[25] M. N. Asl and H. Hosseinzadeh, "Review of pharmacological effects of Glycyrrhiza sp. and its bioactive compounds," Phytotherapy Research, vol. 22, no. 6, pp. 709-724, 2008.

[26] X. Qiao, M. Ye, C. Xiang et al., "Analytical strategy to reveal the in vivo process of multi-component herbal medicine: A pharmacokinetic study of licorice using liquid chromatography 
coupled with triple quadrupole mass spectrometry," Journal of Chromatography A, vol. 1258, pp. 84-93, 2012.

[27] J. Kamei, A. Saitoh, T. Asano et al., "Pharmacokinetic and pharmacodynamic profiles of the antitussive principles of Glycyrrhizae radix (licorice), a main component of the Kampo preparation Bakumondo-to (Mai-men-dong-tang)," European Journal of Pharmacology, vol. 507, no. 1-3, pp. 163-168, 2005. 

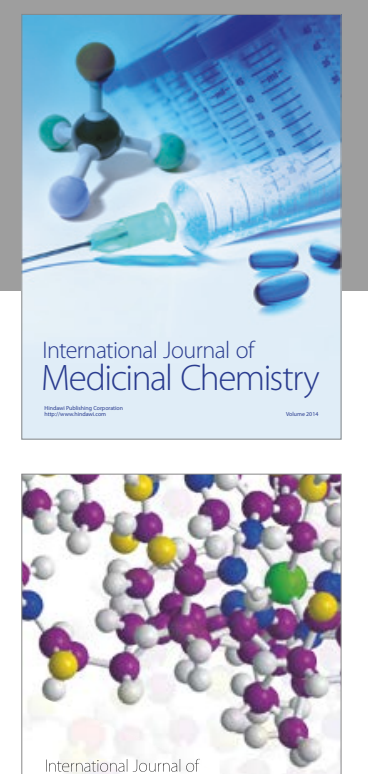

Carbohydrate Chemistry

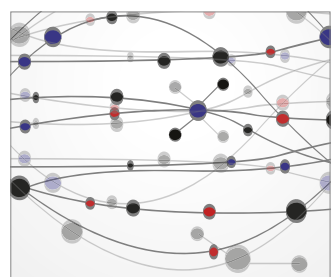

The Scientific World Journal
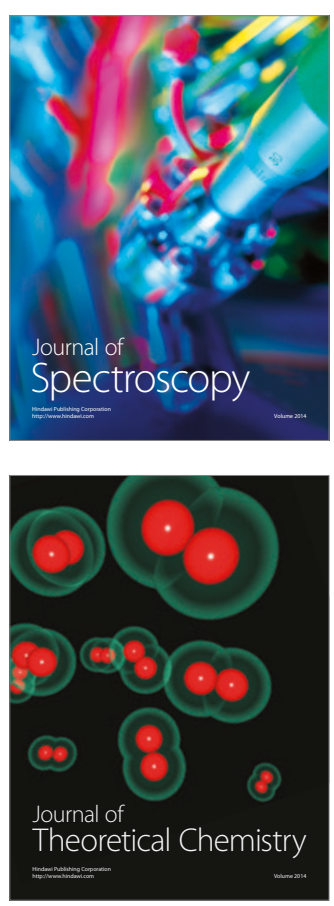
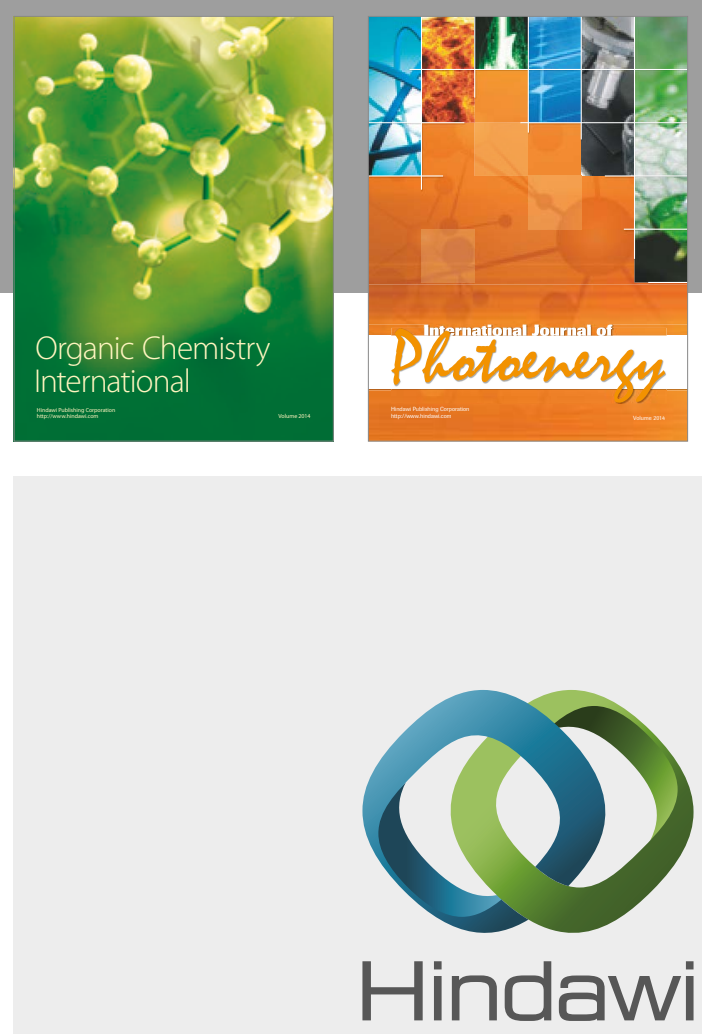

Submit your manuscripts at

https://www.hindawi.com

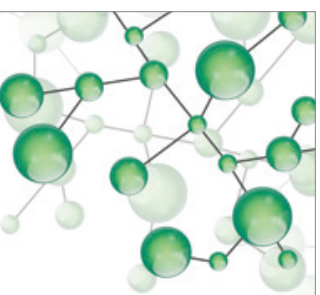

International Journal of

Inorganic Chemistry

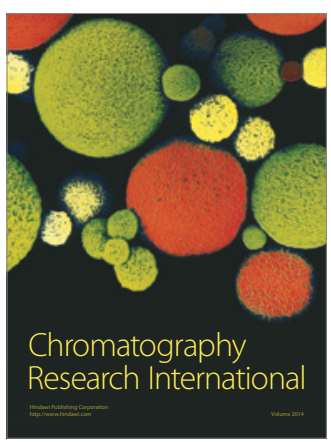

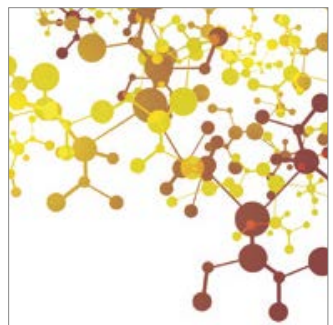

Applied Chemistry
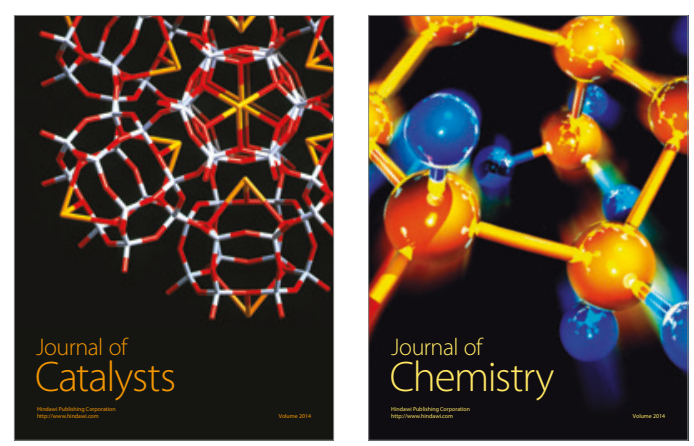
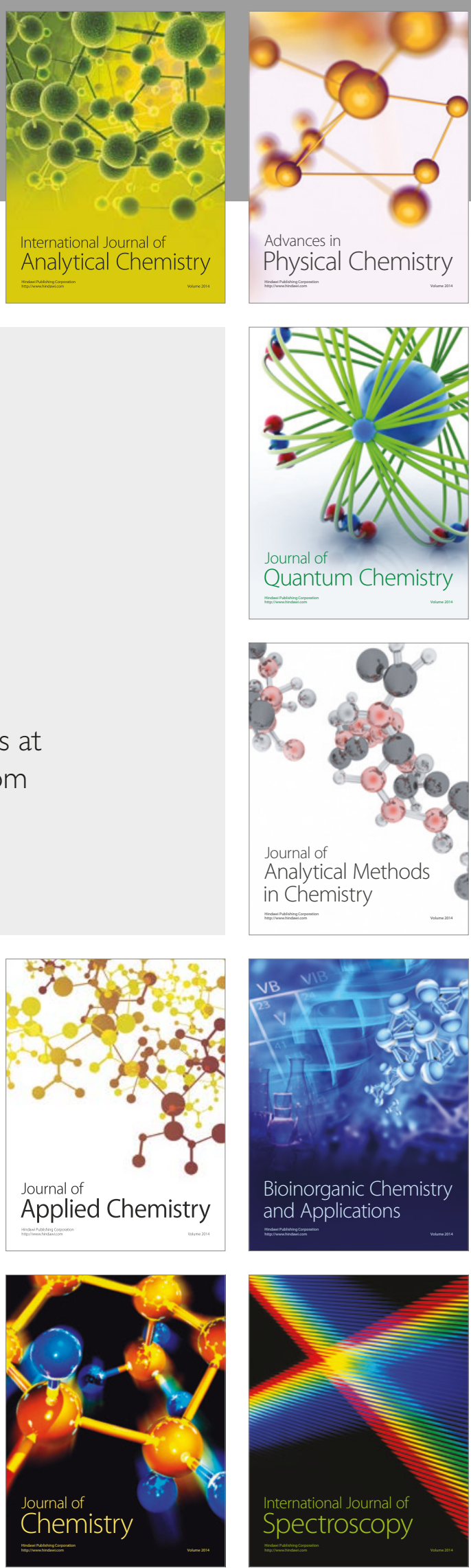